
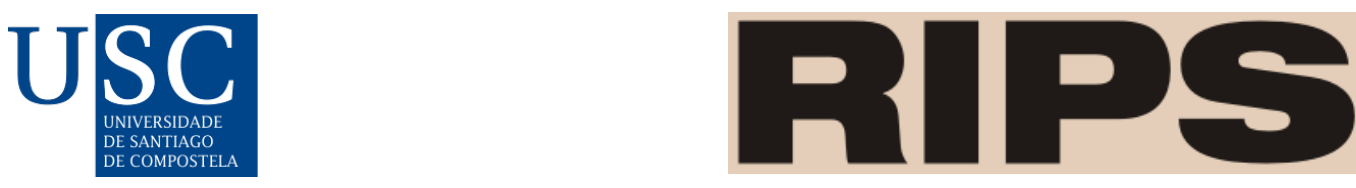

Revista

de Investigaciones

Políticas y Sociológicas

RIPS: Revista de Investigaciones Políticas y Sociológicas, 20(2), 2021. ISSN-e: 2255-5986

https://doi.org/10.15304/rips.20.2.6981

Artículos

\title{
Estudio de mapa de riesgos laborales psicosociales en la Comunidad de Madrid
} Study of a Psychosocial Risks at Work Map in the Community of Madrid

\author{
José Guillermo Fouce Fernandez ${ }^{1, a}$, José Manuel Garcia Bustos ${ }^{2, b}$, Luis García Villameriele \\ ${ }^{1}$ Fundación Psicología Sin Fronteras \\ ${ }^{2}$ Fundación Psicología Sin Fronteras \\ ${ }^{3}$ Fundación Psicología Sin Fronteras \\ a psf@psicologossinfronteras.net ${ }^{b}$ psf@psicologossinfronteras.net ${ }^{c}$ psf@psicologossinfronteras.net
}

\section{Resumen}

Se presenta el primer estudio de mapa de riesgos laborales psicosociales de la Comunidad de Madrid. El objetivo fundamental es situar y describir la presencia e impacto de los riesgos laborales psicosociales en población trabajadora de Madrid. Para ello, se combinaron metodologías cualitativas como estrategias de análisis documental, grupos de discusión o entrevistas a informantes clave junto con metodologías cuantitativas de recogida de datos y procedimientos muestrales. En la novedad de esta investigación por sus características y objeto de estudio, los principales resultados señalan la emergencia de un mercado laboral precario, fraccionado en el que se evidencian de manera particular riesgos laborales como la violencia y la inseguridad contractual. Tales riesgos transforman y cambian aquellos más tradicionales, estableciéndose un escenario complejo para la prevención y la gestión en esta materia. En suma, se hace necesario adaptar las medidas y evaluaciones de Prevención de Riesgos Laborales (PRL), y más concretamente, aquellas dirigidas a riesgos laborales psicosociales, a este nuevo contexto, a la par que desarrollar nuevos modelos de intervención y prevención propiamente dicha.

Palabras clave: riesgos laborales psicosociales; prevención; evaluación.

\begin{abstract}
The development of the first psychosocial occupational risk mapping study carried out in the Community of Madrid is presented and summarised. This research works under the fundamental objective of locating and describing the presence and impact of psychosocial occupational risks in the working population of Madrid. To this end, eminently social methodologies were combined, such as socio-documentary analysis strategies, the dynamization of discussion groups, interviews with key informants, together with data collection methodologies and sampling procedures. The main results point to the emergence of a precarious labour market, in which labour risks such as violence and contractual insecurity are particularly evident. These risks transform and change the more traditional ones, creating a complex scenario for prevention and management in this area. In short, it is necessary to adapt Occupational Risk Prevention (ORP) measures and assessments, and more specifically, those aimed at psychosocial occupational risks, to this new context, as well as to develop new models of intervention and prevention in the strict sense of the term.
\end{abstract}


Keywords: Psychosocial Risks at Work; Labor Risk Prevention; Evaluation.

\section{INTRODUCCIÓN}

El estudio que ahora presentamos. surge de un acuerdo estable de colaboración entre UGT Madrid y la Fundación Psicología Sin Fronteras que durante años han sumado fuerzas para atender a trabajadores y trabajadoras afectados por los riesgos laborales psicosociales, a fin de garantizar su atención integral en lo laboral, jurídico y psicológico. En paralelo, se aborda brindar formación y acompañamiento a los delegados sindicales, en perspectiva de afrontar juntos los retos que plantea el mercado laboral y los riesgos psicosociales.

En este camino de materializar una propuesta de investigación novedosa en la Comunidad de Madrid, presentamos el Primer Mapa de Riesgos Laborales Psicosociales en la Comunidad de Madrid.

Para abordar este reto desarrollamos varios elementos de análisis y construcción:

- $\quad$ En primer lugar, realizamos una amplia revisión bibliográfica sobre la materia y lo que rodea tanto a nivel general como a nivel específico. En general, reunir una rica y variada documentación científica, académica y legal sobre los riesgos laborales y la salud laboral, fue una dinámica central de la búsqueda en clave de investigación.

- Recopilamos un amplio y diverso panel de expertos, del cual extrajimos particularmente, de manera pormenorizada, aquellos que desde muchos prismas se situarían bajo un rol de informantes clave.

- $\quad$ En la parte cualitativa elaboramos un guion de grupos de discusión sobre esta materia, y unas jornadas de presentación cuya función era precisamente de enmarque divulgativo, para posteriormente dinamizar a los asistentes.

- $\quad$ Así mismo desarrollamos un cuestionario/encuesta, que comprende diferentes pruebas y formas de medida validadas (Llorens, Cifre y Nogareda, s.f.; Armangué, Melià y Sesé, 1999; Jorgensen y Schaltz, 2003; González de Rivera y Rodríguez-Abuin, 2005; Fornés, MartínezAbascal y de la Banda, 2008; Blanch, Sahagún, Cantera y Cervantes, 2010; Instituto Nacional de Seguridad y Salud en el Trabajo (INSST), 2018; Pinila, Almodovar, Galiana, Hervas, y Zimmerman, 2018), con las que en conjunto tratamos de obtener las diferentes dimensiones descriptivas del fenómeno de riesgos laborales psicosociales, disponiendo así de una imagen representativamente completa del estado de la situación en la Comunidad de Madrid.

- $\quad$ Por último, y una vez recogidos y triangulados los datos cuantitativos y cualitativos anteriormente señalados, procedemos a desarrollar un informe-memoria de situación con recomendaciones también del camino a seguir.

Como se puede observar, el planteamiento a desarrollar supuso una suma estructurada

de distintas combinaciones y formulas metodológicas constantemente atendidas, acompañadas y revisadas que posteriormente se integraron. Lo cualitativo y cuantitativo se sostenían continuamente. 
Esta suma combinatoria de metodologías fuertemente asentada por datos, visiones y participación de tanto muestra trabajadora como experta en el área, cubrió una serie de objetivos sistemáticos que definieron a la propia naturaleza del mapa a construir:

- Objetivo 1: esta investigación principalmente nace para identificar los riesgos psicosociales existentes en las empresas de la Comunidad de Madrid, o lo que es lo mismo, establecer un diagnóstico del mercado laboral en la Comunidad de Madrid en general y de los riesgos psicosociales en particular.

- $\quad$ Objetivo 2: identificar los principales factores de riesgo psicosocial en las empresas de la Comunidad de Madrid y su incidencia/presencia diferencial por sectores, tipología de empresa, puestos de trabajo y otras variables relevantes.

- Objetivo 3. establecer un Mapa de riesgos psicosociales que permita identificar e implantar medidas preventivas en materia de riesgos psicosociales en las empresas de la Comunidad de Madrid.

\subsection{Antecedentes teóricos de referencia en la práctica}

Volviendo al nivel más macro de la investigación, nuestro mapa de riesgo laboral psicosocial, tomo una fundamentación teórica clara que, si bien replicamos, hubo que adaptar y readaptar constantemente a lo largo del estudio. (Renda, et al., 2017) Los mapas de riesgo son representaciones en cierta manera cartográficas que permiten visualizar la distribución de determinados riesgos de desastre en un territorio específico, y más concretamente en lo que nos correspondía, los riesgos laborales psicosociales insertos en el mercado laboral de la Comunidad de Madrid. Así pues, un mapa de riesgo es, ante todo, una herramienta de análisis esencial que, entre otros usos, permite identificar zonas de mayor o menor riesgo frente a diferentes peligros, como factor clave a la hora de determinar las áreas a intervenir con determinada inversión en infraestructura (Renda, et. al., 2017).

Así, nuestro mapa de riesgos laborales psicosociales tiene por definición la orientación de servir de visión exploratoria y descriptiva de los factores laborales de riesgo psicosociales y los riesgos laborales psicosociales como tal, enmarcándolos en la realidad laboral de un contexto concreto, que en nuestro caso delimitamos en la Comunidad de Madrid.

Hasta aquí, en el momento en el que nos encontrábamos, tomamos varias conceptualizaciones válidas, algunas de ellas fuertemente establecidas temporalmente, otras más actualizadas, empezando por el riesgo laboral que centramos a través de Parra (2003) como aquellos elementos de un trabajo desarrollado que cuentan con la potencialidad de causar un daño.

Teniendo en cuenta el significado per se del riesgo laboral, profundizamos a lo que han significado los factores de riesgo laboral psicosocial, con la definición oficial de la Organización Internacional del Trabajo (OIT) para entrar decididamente al riesgo laboral psicosocial. La OIT define estos factores tan concretos como aquellos que se relacionan con las interacciones entre el contenido y la organización del trabajo, los estilos de dirección, las condiciones organizacionales, por un lado, y las competencias y necesidades de los trabajadores, por otro. Pero ¿qué es exactamente un riesgo laboral psicosocial? En la práctica académica e incluso profesional de la Prevención de Riesgos Laborales (PRL), nos dimos cuenta de que no ha sido fácil ni lo es, responder a esta cuestión, si bien coincidimos que la definición más completa y que salvaguarda la dificultad 
histórica a la hora de distinguir entre riesgo psicosocial y factor psicosocial, refiere a Moreno y Báez (2010, p.17): Un riesgo psicosocial laboral es el hecho, acontecimiento, situación o estado que es consecuencia de la organización del trabajo, tiene una alta probabilidad de afectar a la salud del trabajador y cuyas consecuencias suelen ser importantes. Los riesgos psicosociales, a diferencia de los factores psicosociales, no son condiciones organizacionales sino hechos, situaciones o estados del organismo con una alta probabilidad de dañar la salud de los trabajadores de forma importante. Aunque los riesgos psicosociales impactan en la salud de los trabajadores, los efectos pueden ser diversos y con distinta percepción según el trabajador en su carácter autónomo e individual.

Otro constructo clave divergente y protector ante el riesgo laboral que resultaría y resulta fundamental para el mapa de riesgos es la salud laboral. Hablamos de la definición de salud laboral consensuada por el comité conjunto en Salud Ocupacional formado por la Organización Internacional del Trabajo (OIT) y la Organización Mundial de la Salud (OMS), que se reunió en 1995 con la finalidad de abarcar tal concepto.

De la declaración de la OIT y la OMS (1995) se consensua que la salud laboral es una orientación de acciones en contexto de trabajo, dirigidas a la promoción y el mantenimiento más alto de bienestar físico, psicológico y social del personal trabajador en todas las ocupaciones; la prevención en los trabajadores de las alteraciones de la salud causadas por sus condiciones de trabajo; la protección de los trabajadores del riesgo resultante de los factores adversos a la salud; la ubicación y el mantenimiento de los trabajadores en un ambiente adaptado a sus capacidades fisiológicas y psicológicas, y la adaptación del trabajo al trabajador y de cada trabajador a su trabajo (MorenoJiménez y Garrosa, 2013). La salud laboral es un proceso en sí mismo que contempla el riesgo como una posibilidad de importante calado en los ambientes de trabajo, y que implica acciones específicas de manera comprometida y proactiva para proteger al personal trabajador y su contexto.

En este trabajo de mapa de riesgos concebimos la salud laboral ante los riesgos laborales psicosociales como una cultura corporativa en todo su significado, una manera de pensar y actuar generalizada, basada en el respeto, el cuidado y preocupación por la salud como conductas de la organización (Schein, 1988), hablamos de una cultura de la seguridad laboral basada en valores (Barling y Frone, 2004) más allá de protocolos estandarizados o manualizados que basan sus técnicas en evitar la amenaza para la salud. El cambio está en sustituir la reacción ante la amenaza de riesgo (evitar el accidente y la enfermedad profesional, disminuir la baja laboral por problemas de salud y facilitar la integración rápida al trabajo), en vigilancia de la salud que evite cualquier daño, y utilizarlo como un medio para aumentar el capital físico, humano y social de la organización (desarrollo físico, social y mental de los trabajadores) (Black, 2008; Härtel y Ashkanasy, 2011; Moreno-Jiménez y Garrosa, 2013). Para ello, consideramos clave concebir la salud laboral como objetivo estratégico de la misma empresa y a sus valores de base, tal y como desarrollan MorenoJiménez y Garrosa (2013), pero, además, contemplar una participación cohesionada que tome en cuenta la responsabilidad compartida en este sentido, frente a los riesgos laborales psicosociales.

\subsection{La actualidad teórica de los riesgos laborales psicosociales en la práctica}

En el momento en que este artículo se redacta, el estudio de los riesgos laborales psicosociales ha continuado bajo perspectivas diversas, si bien consideramos que hacen falta más acercamientos a la 
luz de trabajos de revisión documental como el de Rivera-Porras (2019) o Salamanca et al. (2019), sobre todo en nuestro país.

Destacando el trabajo de Salamanca et al. (2019), se ahonda en el efecto de los factores de riesgo laboral psicosocial dentro del fenómeno conjunto de riesgo laboral, captando mayormente las consecuencias que implican en visión del personal trabajador. Tras una exhaustiva búsqueda documental y selectiva, la conclusión es similar al trabajo citado de Rivera-Porras (2019). El factor de riesgo incide como una interacción problemática entre el trabajo y el ambiente laboral, como resultado de un esfuerzo de trabajadores y trabajadoras por adaptarse a las demandas del entorno. Muy importante el reconocimiento que Salamanca et al. (2019) extraen en la línea de transitar a un enfoque sistémico que integre todos los elementos del ambiente laboral que supongan convertirse en factor de riesgo en tres niveles: el individuo, el trabajo y el entorno (Arenas y Andrade, 2013). En estos términos, comprender también la experiencia del trabajador como una vivencia activa, sujeta a contingencias ambientales que tanto puedan ser protectoras de su salud, como implicar daños ya no solo en el nivel individual, sino a escala multinivel. Captamos en esta cuestión una responsabilidad por tanto no solo impuesta al personal trabajador, sino interactiva y proporcionalmente participante en lo global.

Aún en la imperante necesidad documental de España en estos términos, observamos que han tenido lugar trabajos esencialmente significativos como el análisis de Ballesteros et al. (2019) que visibiliza la enorme incidencia de estos riesgos en personas con trastorno mental grave. De base, esta población cuenta con mayores factores de vulnerabilidad a sufrir riesgos laborales psicosociales (Sobrino, 2001; Perkins y Rinaldi, 2002; López et al., 2010), y particularmente manifiestan percibir ante determinados puestos de trabajo un control con horarios y cuadrantes muy rígidos, donde la tarea no se adapta a las circunstancias y momentos personales (Ballesteros et al., 2019).

El más reciente trabajo de Henry (2019), en un ejercicio de evidencia significativo, explica el proceso incidental de los riesgos laborales psicosociales en el tejido productivo-laboral de Argentina. Los grupos con mayor situación contextual de vulnerabilidad socioeconómica son también los más expuestos a convertirse en un precio humano de la expresión específica de la acumulación capitalista en lo laboral. El análisis exhaustivo de esta fuente documental nos invita a no solo captar única y exclusivamente la dimensión individual en el riesgo laboral psicosocial, sino a entender lo individual también como una dinámica integral interdependiente con lo colectivo.

\section{METODOLOGÍA Y ANÁLISIS DATOS}

Desarrollamos un análisis pormenorizado, descriptivo, preciso y flexible. Se recogieron datos por varias vías, combinando lo cuantitativo con lo cualitativo.

a) Con respecto a la revisión bibliográfica general y especifica: o lo que es lo mismo, el análisis documental, giró en torno a tres ejes marcadamente estructurados.

a. Análisis desde la base de datos en español Psicodoc y la biblioteca del Colegio Oficial de Psicólogos de Madrid (COPM).

b. Estudio documental basado en análisis bibliográfico genérico en torno a áreas temáticas diversas.

c. Recopilación de fuentes normativo-legales en la materia. 
b) Con respecto a las entrevistas a expertos con guion de entrevistas semi estructuradas. Se seleccionaron agentes clave, informantes y fundamentales para tener distintas miradas de lo que acontece en el mercado laboral.

c) Con respecto a los grupos de discusión. Si bien, siguió un modelo muy estructurado, las personas tenían turnos de palabra, podían expresarse de una manera mucho más abierta, aunque protocolarizada. Participaron en grupos unas 120 personas. En esta muestra el perfil mayormente presente en los grupos fue la figura de trabajador y delegado sindical, que de hecho conformaron 3 de los 4 grupos establecidos. El acceso a esta muestra supuso convocar mediante Jornadas divulgativas desde UGT-Madrid y Psicología Sin Fronteras a los asistentes de manera generalizada resultando en un éxito de asistencia.

d) Con respecto al cuestionario desarrollado por el equipo de investigación, el Cuestionario de Riesgos Psicosociales 2019: siguió el sistema Computer Assisted Telephone Interviewing (CATI) (Agudelo, et al., 2010). Subyaciendo extensos esfuerzos con pruebas resultantes, que miden distintos tipos de riesgo laboral, factores organizacionales y laborales, entre otros, que están establecidos en la práctica de la identificación y evaluación propias de la Prevención de Riesgos Laborales. Se desarrolló un muestreo aleatorizado, y dirigido por vía telefónica a trabajadores de la Comunidad de Madrid con carácter sociodemográfico diverso. En total, 1000 personas con edades comprendidas entre los 18 y 65 años, repartidas en cuotas por sexo y edad proporcionales a la población censada en la Comunidad de Madrid y que en el momento del estudio estaban trabajando. En adelante, comentamos en detalle las propiedades de cada una de las pruebas utilizadas para construir el cuestionario final:

- $\quad$ Cuestionario en español de la 6 ${ }^{a}$ Encuesta Europea de Condiciones de Trabajo (Pinila, Almodovar, Galiana, Hervas, y Zimmerman, 2018): construido para población en edad legal de trabajar (en España, 16 años es la edad mínima). Esta herramienta eminentemente descriptiva, permitió extraer las condiciones de vida y laborales de la población europea, y particularmente de España. Está planteado para aplicarse de manera entrevistada en un tiempo medio de 45 minutos. El cuestionario consta de aproximadamente 107 ítems repartidos en varias secciones categoriales (condiciones de empleo; exposición a riesgos físicos, ambientales y ergonómicos; riesgos psicosociales y organizativos; actividad preventiva y organización; tiempo de trabajo y conciliación; formación; salud y bienestar).

- Instrumento de valoración de riesgos psicosociales del INSST (FPSICO) (Version 4.0) (Instituto Nacional de Seguridad y Salud en el Trabajo; INSST, 2018): se trata de una herramienta de evaluación en riesgos laborales psicosociales, diseñada para utilizarse, adecuando a las características de la organización, y a los objetivos para los que se utilice, con los recursos disponibles. Se puede aplicar mediante una aplicación informática, y consta de 89 ítems distribuidos en 9 factores diferenciados: tiempo de trabajo, autonomía, carga de trabajo, demandas psicológicas, variedad/contenido del trabajo, participación/supervisión, interés por el trabajador/compensación, desempeño de rol, y relaciones y apoyo social. 
La consistencia interna del instrumento asciende a un coeficiente $\alpha$ de Cronbach de 0,895 .

- Método ISTAS (CoPsoQ), instrumento para la prevención de riesgos psicosociales, versión corta para pequeñas empresas y autoevaluación (Versión 21) (Armangué, Jorgensen y Schaltz, 2003): al igual que la prueba anterior, este instrumento permite evaluar riesgos laborales psicosociales, en este caso, enfocando a pequeñas empresas (menos de 30 trabajadores). Consta de 39 ítems, distribuidos en varias dimensiones: doble presencia; exigencias psicológicas cuantitativas; exigencias emocionales; exigencias de esconder emociones; influencia; Posibilidades de desarrollo en el trabajo; Control sobre los tiempos de trabajo; Sentido del trabajo; Integración en la empresa; Previsibilidad; Claridad de rol; Calidad de liderazgo; Apoyo social en el trabajo; Posibilidades de relación social; Sentimiento de grupo; Inseguridad en el trabajo; Estima. La consistencia interna del instrumento asciende a un coeficiente $\alpha$ de Cronbach de entre 0,65 hasta 0,92 , valores por ende fiables y adecuados para una prueba de estas características.

- Cuestionario de acoso psicológico percibido (Morán, González y Landero, 2009): esta prueba permite evaluar el sentimiento de acoso psicológico en el lugar de trabajo. Consta de un formato breve de 15 ítems en autoinforme. La consistencia interna del instrumento asciende a un coeficiente $\alpha$ de Cronbach de 0,92 .

- $\quad$ Cuestionario de hostigamiento psicológico en el trabajo (Fornés, MartínezAbascal y de la Banda, 2008): el presente cuestionario mide en efecto el hostigamiento psicológico en el trabajo, es decir, la frecuencia del acoso a través de una escala de 5 puntos. Este instrumento dispone 35 ítems con cinco factores principales: Humillación y rechazo personal, Desprestigio profesional, Rechazo profesional y violación de la intimidad, Degradación profesional y Ninguneoaislamiento profesional. La consistencia interna del instrumento asciende a un coeficiente $\alpha$ de Cronbach de 0,96.

- $\quad$ Cuestionario de estrategias de acoso en el trabajo. El LIPT-60 (González de Rivera y Rodríguez-Abuin, 2005): esta prueba proporciona una perspectiva global en la frecuencia e intensidad percibidas de las estrategias de acoso laboral de quien las padece, mediante 60 ítems organizados en 6 subescalas: Subescala de desprestigio laboral (DL), Subescala de entorpecimiento del progreso (EP), Subescala de incomunicación o bloqueo de la comunicación (BC); Subescala de intimidación encubierta (IE); Subescala de intimidación manifiesta (IM); Subescala de desprestigio personal (DP). La consistencia interna del instrumento asciende a un coeficiente $\alpha$ de Cronbach de entre 0,83 a 0,94 entre todas las subescalas.

- Cuestionario de bienestar laboral general (Blanch, Sahagún, Cantera y Cervantes, 2010): este cuestionario permite evaluar principalmente el bienestar psicológico laboralmente situado en su circunstancia mediante dos grupos de escalas. En el primero se obtiene información de bienestar psicosocial y está compuesto por 42 ítems repartidos en tres factores: las escalas de Afectos (10 ítems), de Competencias (10 ítems) y de Expectativas (22 ítems). En el segundo 
grupo de escalas, se obtiene información sobre efectos colaterales mediante 13 ítems, repartidos en tres factores: las escalas de Somatización (5 ítems), de Desgaste (4 ítems) y de Alienación ( 4 ítems), en total con los dos grupos de escalas, la prueba dispone 55 ítems.

- $\quad$ Cuestionario de Tecnoestrés (Tecnoansiedad y Tecnofatiga; Llorens, Cifre y Nogareda, s.f.): prueba con formato de cumplimentación online y físico con escala de frecuencia. Permite medir el tecnoestrés como daño psicosocial mediante tres escalas: 1) Afectiva (ansiedad vs. fatiga), 2) Actitudinal (actitud escéptica hacia la tecnología) y 3) Cognitiva (creencias de ineficacia en el uso de la tecnología). Está dirigido específicamente a trabajadores que utilizan TICs en su trabajo. Puntuaciones altas en las tres escalas suponen indicadores de tecnoestrés en dos manifestaciones: 1) tecnoansiedad (altas puntuaciones en ansiedad, escepticismo e ineficacia) y 2) tecnofatiga (altas puntuaciones en fatiga, escepticismo e ineficacia). La consistencia interna del instrumento asciende a un coeficiente $\alpha$ de Cronbach de entre 0,82 a 0,92 entre las tres escalas en población general y en usuarios de tecnología.

- $\quad$ Cuestionario C3/15 (Melià y Sesé, 1999): con este instrumento se logra extraer el clima organizacional. Aquel determinado operativamente como la percepción del trabajador acerca del conjunto de acciones hacia la seguridad realizadas por la empresa, con efectos sobre la conducta del personal, tanto a nivel de planificación y organización, como de intervención y mejora. El cuestionario se distribuye en 15 ítems repartidos en una estructura trifactorial: estructuras de seguridad, la política de la empresa en seguridad y las acciones de intervención en seguridad puestas en práctica por la empresa. La consistencia interna del instrumento asciende a un coeficiente $\alpha$ de Cronbach de 0,87 .

\section{RESULTADOS}

De las entrevistas a informantes clave:

- Hay constancia de que el empleo ha vivido un cambio a nivel social significativo desde los años en los que incidía notablemente la crisis económica. Se ha convertido en un bien inseguro y no garantizado. Un valor únicamente instrumental desde el que conseguir beneficios, salario, perdiéndose la identidad personal de quien lo desempeña y con la propia actividad en sí.

- $\quad$ Se coincide en que el trabajo como un medio de realización personal ha desaparecido para dar paso como resistencia a un contexto hostil.

- $\quad$ Existe una pérdida de conceptualización de los contenidos de trabajo. La precariedad viene también expresada en confusión de contenido entre tareas y funciones en bastantes puestos de trabajo.

- $\quad$ En suma, con lo previo, nuestros expertos se postulan en señalar también la precariedad como baja remuneración, contratos laborales inseguros, y en concreto aquellos a tiempo parcial. Los contratos temporales del mismo modo se han incrementado. 
- $\quad$ Las personas mayores de 45 años son principales víctimas de los riesgos laborales psicosociales y constan de la dificultad añadida del acceso al empleo.

- Los expertos ven en las características del mercado laboral adjetivos tales como incertidumbre, inseguridad y cortoplacismo, siendo representativos en la medida en que suponen mayor evidencia de precariedad.

- Los riesgos laborales psicosociales que nuestros informantes otorgan consenso en un amplio espectro de sectores y territorios son: inseguridad contractual, devaluación salarial, desigualdad económica y de condiciones generales entre los trabajos en función de los deciles económicos respectivos.

- Los expertos que otorgan distinción en como un riesgo psicosocial se puede expresar de acuerdo a los sectores productivos reconocen que en los sectores con más presencia femenina se ven ellas más expuestas al acoso psicológico, sexual, destacando el sector agrícola. En los sectores menos cualificados el impacto de estos riesgos es mayor. Hay bastante proximidad de posturas cuando se trata de apuntar en los casos de violencia. Estos están presentes en sectores de cara al público, mayormente el sector servicios. En conclusión, los informantes se postulan a enfatizar que sin duda el estrés es el riesgo mayormente presente en todos los sectores sin diferencia entre cada uno, sobre todo en el sector industrial, se caracteriza especialmente.

- $\quad$ Algunos de los nuevos riesgos psicosociales y emergentes que tienen especial cabida entre nuestros expertos, seleccionando de hecho algunos ya referidos están:

- $\quad$ La pérdida del marco de desarrollo de carreras.

- $\quad$ La intensidad y nivel de exigencias incremental requerido.

- $\quad$ La conexión con el trabajo 7/24, siete días a la semana durante 24 horas.

- $\quad$ La GIG economía y las plataformas digitales de empleo junto con sus condiciones de trabajo derivadas.

- $\quad$ Las nuevas relaciones de trabajo en el marco de las TIC y la IA.

- La realidad en suma a la que finalmente los informantes infieren, es que se hacen pocas identificaciones y evaluaciones de riesgos psicosociales. La mayoría son simplemente reactivas a alguna campaña. De forma proactiva, solo cuando hay un certificado o lo pide la inspección de trabajo. Por otra parte, las metodologías son adecuadas y las estrategias igual, lo que ocurre es que no se hacen con la frecuencia o profundidad que se deberían hacer, de manera preventiva y proactiva.

En segundo lugar, los resultados que se delimitan de los grupos de discusión en su totalidad suponen de nuevo varios consensos significativos:

- $\quad$ Desde el nivel de empresa, las primeras ideas que se abren paso tienen que ver con una voluntad y necesidad general de concienciar en prevención de riesgos laborales, dado que, en la práctica, el cumplimiento de esta dinámica se limita a cubrir expediente o en los supuestos en los que media una sanción de la inspección de trabajo. Coinciden en que dentro del sector servicios la prevención de riesgos se percibe con inseguridad. Del desinterés general desde el punto de vista de organización, se suma la lentitud con que inspección de trabajo se pronuncia al respecto de los riesgos psicosociales. La principal víctima en consecuencia es el trabajador. 
- Los grupos son conscientes de que el estrés es el gran riesgo, el más presente, y como tal, podría intervenirse con la correcta actuación en los factores de riesgo.

- Ampliando en la línea de riesgos psicosociales que los grupos visibilizan encontramos:

- $\quad$ Precariedad (ejemplos: contratos por obra y los bajos salarios). Añadiéndose una situación de miedo actual frente a estas circunstancias.

- $\quad$ Trabajar muchas horas con muy baja remuneración, pero, además, a veces tienes un status en la empresa y aun así estás haciendo un trabajo que no te corresponde y no se te remunera. De hecho, las horas que se trabajan de más no están remuneradas, y paralelamente también repercute en menos conciliación familiar.

- $\quad$ Dificultad de conciliación vida familiar y ocio con trabajo.

- $\quad$ Presión, sobrecarga y el hecho de que se haya disminuido el personal en muchas empresas, pero no el volumen de trabajo.

- Los grupos detectan que los departamentos que debieran velar por el cuidado y protección del trabajador, véase: los departamentos de quejas, éticas, RRHH no ayudan en nada por mero posicionamiento. Existen muchas situaciones de acoso en las empresas que no pueden probarse en favor del trabajador a razón de esta parcialidad, por tanto, se coinciden en que se necesita de figuras imparciales y objetivas.

- Hay ciertas posturas que se enfatizan en afirmar concienzudamente que no existen estrategias de identificación/evaluación de riesgos psicosociales. Además, establecen crítica en la que inciden en reconocer que además la empresa no quiere verlo, porque económicamente la organización justifica encubiertamente que es inviable dar bajas por estrés.

En tercer lugar, cuanto deviene en los resultados cuantitativos, observamos a continuación que destacan algunos de los siguientes:

- $\quad$ Las diferencias de género por ejemplo en la temporalidad de los contratos, algo que afecta el doble a las mujeres que a los hombres.

- Las diferencias también en cuanto a la edad siendo así las mujeres jóvenes las que se encuentran en peor situación y presentan una mayor vulnerabilidad.

- Se encuentran datos que vuelven a respaldar la brecha económica entre mujeres y hombres, sumado además a que los jóvenes y las mujeres manifiestan mayor insatisfacción con el trabajo, constatándose al mismo tiempo, amplias diferencias entre trabajadores autónomos o asalariados en diferentes situaciones. Los jóvenes de hecho se sienten más discriminados con respecto al resto de los grupos.

- Hay amplios rasgos de variación en materia de satisfacción con el trabajo con muchas divergencias, los hombres se muestran más satisfechos que las mujeres y la satisfacción aumenta con la edad de los trabajadores. Con ello, aparecen efectos emocionales significativos del trabajo cuando los situamos en términos de condiciones laborales y cultura organizacional, por ejemplo: se manifiesta preocupación ante los supuestos de cambio de salario o de horario de trabajo, también por posibles cambios de trabajo. Las mujeres, al respecto, se muestran más preocupadas que los hombres.

- $\quad$ Existiendo valores significativos de estrés en los trabajadores, hay percepción de que el trabajo afecta de forma negativa a su salud, la intensidad que atribuyen a los problemas de salud que se manifiestan es escasa. 
- $\quad$ Solo el 29,3\% de los encuestados piensa que se han realizado alguna vez en su empresa la evaluación de los riesgos psicosociales, algo incluso mucho menor en los autónomos.

- $\quad$ Se observa que, aunque el salario de los hombres es ligeramente superior al de las mujeres, los hombres trabajan más horas a la semana que las mujeres (un mayor porcentaje de mujeres que de hombres tiene turno continuo fijo), ya sea por las preferencias de las mujeres o para facilitar la conciliación laboral (son las que más horas dedican a las tareas del hogar y a las actividades relacionadas con la atención y cuidado de los hijos y/o nietos). Sin embargo, los hombres pueden dejar con mayor frecuencia su puesto de trabajo, al menos una hora, sin pedir un permiso especial debido a un asunto personal o familiar, están menos expuestos a los cambios en los horarios de trabajo.

- $\quad$ Ante esta situación, las mujeres describen estar más preocupadas por los cambios de su salario o de su horario de trabajo, siendo también las más críticas en cuanto a la cobertura de las necesidades materiales y de desarrollo personal. En relación con estas preocupaciones, si bien a los más jóvenes también les preocupa la cobertura de sus necesidades de desarrollo personal, la preocupación por quedarse en el paro se incrementa a medida que aumenta la edad de los individuos. Con todo, el grado de satisfacción con la empresa es mayor entre los hombres que entre las mujeres, aumentando con la edad de los entrevistados.

\section{DISCUSIÓN Y LIMITACIONES}

En primer lugar, cabe señalar la dificultad del estudio desarrollado en tanto existen pocos o insuficientes referentes anteriores con los que comparar o contrastar. Así mismo, resultó complejo por la extensión y variedad del propio objeto de análisis, en contexto de mercado laboral, con muy diferentes estratos y situaciones. De cualquier manera, todo el estado del arte recogido resultó enormemente decisivo.

Resultó complicado descubrir y señalar la emergencia de nuevos riesgos psicosociales que aparecen en el entorno laboral, tal y como reflejaron nuestros expertos, como los datos cualitativos que a cambio pudimos recoger, que siempre dan una mayor profundidad a lo que investigamos. Por ejemplo, la violencia laboral, o acoso en múltiples variantes, sobre todo, acoso sexual, aparece como un gran reto de analizar y medir. Creemos, que en lo que comprende al mapa de riesgos, violencia y acoso, no han quedado correctamente descritos en la muestra. Atribuimos varias causas, siendo el efecto del método, una de ellas. En concreto, lo cuantitativo y en general, los diseños de investigación siendo directivos y en cierta manera espontáneos e inmediatos, pueden resultar invasivos dada la naturaleza de la información que se obtiene, que muchas veces es significativamente sensible. A grosso modo, sesgos como: el sesgo por subjetividad, entre otros, quedan contemplados como posibilidad en el mapa. Pero especialmente, hay consenso en hipotetizar Deseabilidad Social (DS). Desde lo que corresponde al mapa creemos que la DS, ha tenido impacto muy sustancialmente en lo cuantitativo como tendencia a que los participantes muestren una imagen favorable de ellos mismos con respecto a las empresas en las que trabajan, y/o directamente de sus empresas.

Hasta aquí, proponemos en particular, que en futuras encuestas que se desarrollen haya un mayor énfasis en crear marcadores fiables y válidos de sesgos, y al mismo tiempo, valorar nuevas opciones de recogida de datos para el entrevistado. Si se desea replicar esta investigación, derivar hacia constructos más específicos. 
Necesitamos abordar de manera compartida entre todos los agentes implicados en este particular: administración, sindicatos, empresa y trabajadores y trabajadoras, en definitiva, voluntad de todos y todas, desde la realidad social, pero también legal y política. A este respecto, resulta además sujeto a mejora, la ampliación de participación de empresarios y las mutuas que tienen poca o nula representación en la muestra.

De todo, establecemos que hay evidencias descriptivas de un mercado laboral marcado por elevadas tasas de precariedad y temporalidad, con una profunda transformación del significado del trabajo que, en no pocas ocasiones, deja de ser el eje vertebrador para la inclusión social y para la construcción de la identidad personal ya que se produce la fuerte emergencia y constatación de la existencia de trabajadores pobres, o de un grupo extenso de lo que viene en denominarse "precariado" con muchas rotaciones en puestos de trabajo, mucha temporalidad e inestabilidad.

\section{CONCLUSIONES}

Entre las conclusiones sucesivas a cuanto contemplamos de este artículo, consideramos cabe señalar las siguientes:

- La necesidad de replicar y darle continuidad a un trabajo de este tipo para, a modo de observatorio más o menos permanente, conocer la evolución de los datos recogidos y del propio mercado laboral en secuencias de datos que nos permitan medir y evaluar la situación, planteando claves de intervención sobre la situación.

- La existencia de delegados sindícales, la evaluación de riesgos y los planes de prevención de riesgos son factores que mejoran la situación. Resulta fundamental que se realicen evaluaciones para la prevención de los riesgos psicosociales, pues cuentan con una baja instauración en las empresas a la luz de los resultados, especialmente, en las de los autónomos.

- $\quad$ Recalcamos que el riesgo psicosocial tiene que empezar cambiando en el gobierno entendiéndose este como "brazo ejecutor" de iniciativas legales nuevas, y acordes con la necesaria intervención bajo un nuevo paradigma, más consciente y sensible con esta problemática. Para que las empresas puedan tomar conciencia y ejemplo, este cambio se debe materializar a través de las administraciones.

En razón de resumir aquellas obtenidas desde el propio mapa de riesgos a efectos divulgativos, aportamos a continuación en primer lugar aquellas más relevantes, referentes a nuestros informantes, pero antes de ello, visibilizamos nuestra perspectiva sintética y contextual de cuanto muestra nuestro mapa de riesgos:

- $\quad$ Cultura de lo inmediato en una disponibilidad laboral constantes, donde el trabajador tiene miedo ante la precariedad propiamente dicha.

- $\quad$ Trabajar horas y horas, siendo parte de ellas con baja y/o ninguna remuneración. En consecuencia, trabajar con confusión de tareas, y con menos conciliación vida-trabajo.

- $\quad$ Presión, sobrecarga, y disminución de plantillas, en contraposición al aumento de volumen de trabajo.

- Deshumanización e individualidad de las condiciones laborales, con climas de competitividad y desidentificación profesional. 
- Carencia de negociación colectiva e incomunicación empresa-trabajador, repercutiendo en inacción sobre los riesgos laborales psicosociales.

- Vulneración de derechos de inserción laboral en multitud de perfiles, con el añadido de inmovilidad de las empresas ante la promoción y desarrollo del personal trabajador senior.

A continuación, nuestros informantes:

- $\quad$ El empleo ha vivido un cambio a nivel social significativo desde los años en los que incidía notablemente la crisis económica (2008-2012). Se ha convertido en un bien inseguro y no garantizado. Un valor únicamente instrumental.

- Los expertos ven en las características del mercado laboral adjetivos tales como incertidumbre, inseguridad y cortoplacismo, siendo representativos en la medida en que suponen mayor evidencia de precariedad.

- La realidad en suma a la que finalmente los informantes infieren, es que se hacen pocas identificaciones y evaluaciones de riesgos psicosociales. La mayoría son simplemente reactivas a alguna campaña. De forma proactiva, solo un $10 \%$ aprox. realizan estas evaluaciones. Solo cuando hay un certificado o lo pide la inspección de trabajo.

En segundo lugar, compartimos las conclusiones cuantitativas a destacar:

- $\quad$ Existen diferencias de género significativas en la temporalidad de los contratos, siendo algo que afecta el doble a las mujeres que a los hombres. También en cuanto a la edad siendo así las mujeres jóvenes las que se encuentran en peor situación y presentan una mayor vulnerabilidad.

- $\quad$ Se encuentran datos que vuelven a respaldar la brecha económica-laboral entre mujeres y hombres, sumado además a que los jóvenes y las mujeres manifiestan mayor insatisfacción con el trabajo, constatándose al mismo tiempo, amplias diferencias entre trabajadores autónomos o asalariados en diferentes situaciones. Los jóvenes de hecho se sienten más discriminados con respecto al resto de los grupos.

- Hay amplios rasgos de variación en materia de satisfacción con el trabajo con muchas divergencias, los hombres se muestran más satisfechos que las mujeres y la satisfacción aumenta con la edad de los trabajadores.

- $\quad$ Resulta fundamental que se realicen evaluaciones para la prevención de los riesgos psicosociales, pues cuentan con una baja instauración en las empresas a la luz de los resultados, especialmente, en las de los autónomos.

\section{Bibliografía}

AGUDELO, G., RUIZ RESTREPO, J. Y AIGNEREN, M. (2010). Metodología de las encuestas telefónicas. La Sociología en sus Escenarios, (21).

ARENAS, F. y ANDRADE, V. (2013). Factores de riesgo psicosocial y compromiso (engagement) con el trabajo en una organización del sector salud de la ciudad de Cali, Colombia. Revista Acta Colombiana de Psicología, 16(1). 43-56.

BALlESTEROS, F., VAQUERO, C., VITUTIA, M., MOLINERO, C., LARIOS, M., DELGADO, V., CALVO, R. (2019). Análisis de la percepción de riesgos psicosociales en personas con trastorno mental grave en el contexto laboral. Clínica Contemporánea, 10, e11,. https://doi.org/10.5093/cc2019a11 
BARLING, J. y FRONE, M. R. (2004). The Psychology of Workplace Safety. Washington, DC: American Psychological Association.

BLACK, C. (2008). Working for a healthier tomorrow: A review of Britain's working age population. Norwich: The Stationery Office.

CROWNE, D. P., \& MARLOWE, D. (1960). A new scale of social desirability independent of psychopathology. Journal of Consulting Psychology, 24(4), 349-354.

CUESTIONARIO C3/15 extraído de: Melià, J. L. y Sesé, A. (1999). La medida del clima de seguridad y salud laboral. Anales de Psicología/Annals of Psychology, 15(2), 269-289.

CUESTIONARIO DE ACOSO PSICOLÓGICO percibido extraído de: Morán, C., González, M. T., y Landero, R. (2009). Valoración psicométrica del cuestionario de acoso psicológico percibido. Revista de Psicología del Trabajo y de las Organizaciones, 25(1), 7-16.

CUESTIONARIO DE BIENESTAR LABORAL GENERAL extraído de: Blanch, J. M., Sahagún, M., Cantera, L. y Cervantes, G. (2010). Cuestionario de bienestar laboral general: estructura y propiedades psicométricas. Revista de Psicología del Trabajo y de las Organizaciones, 26(2), 157-170.

CUESTIONARIO DE HOSTIGAMIENTO PSICOLÓGICO EN EL TRABAJO extraído de: Fornés, J., MartínezAbascal, M. A. y de la Banda, G. G. (2008). Análisis factorial del cuestionario de hostigamiento psicológico en el trabajo en profesionales de enfermería. International Journal of Clinical and Health Psychology, 8(1), 267-283.

CUESTIONARIO DE TECNOESTRÉS (Tecnoansiedad y Tecnofatiga) extraído de: Llorens, S., Cifre, E. y Nogareda, C. (s.f.) NTP 730: Tecnoestrés: concepto, medida e intervención psicosocial.

CUESTIONARIO EN ESPAÑOL DE LA 6 ${ }^{\mathrm{a}}$ ENCUESTA EUROPEA DE CONDICIONES DE TRABAJO extraído de: Pinila, G. J., Almodovar, M. A., Galiana, B. M., Hervas, R. P., y Zimmerman, V. M. (2018). Encuesta Nacional de Condiciones de Trabajo. 2015 6 ${ }^{a}$ EWCS-España. Instituto Nacional de Seguridad e Higiene en el Trabajo (INSHT).

EIJKEMANS, G. (2003). El programa de Salud ocupacional de la Oficina central de la Organización Mundial de la Salud (OMS). The Global Occupational Health Network.

GARCÍA BUSTOS, J.M., FOUCE FERNÁNDEZ, J.G. y GARCÍA VILLAMERIEL, L. (2019). In UGT-Madrid (Ed.), I Mapa de Riesgo Laboral Psicosocial en la Comunidad de Madrid (1ํㅡ ed.). Madrid, España: Gráficas de Diego.

HARTEL, Ch. E. J. y ASHKANASY, N. M. (2011). Healthy Human Cultures as Positive Works Environments. En N. M. Ashkanasy, C. P. M. Wilderom y M. F. Peterson (eds.), The Handbook of Organizational Culture and Climate (pp. 85-100). Thousand Oaks, CA: Sage Publications.

HENRY, M. L. (2019). Salud laboral en el escenario productivo actual: la creciente incidencia de los riesgos psicosociales. Revista de Ciencias Sociales, 32(44), 171-196. Epub 01 de junio de 2019. https://dx.doi .org/10.26489/rvs.v32i44.8.

INSTITUTO NACIONAL DE SEGURIDAD Y SALUD EN EL TRABAJO (INSST) (2018). Instrumento de valoración de riesgos psicosociales del INSST (FPSICO) (Version 4.0).

LÓPEZ, M., LAVIANA, M. y GONZÁLEZ, S. (2010). Rehabilitación laboral y programas de empleo. En A. Pastor, A. Blanco y D. Navarro (2010). (Dirs.). Manual de rehabilitación del trastorno mental grave (pp. 511-537). Madrid, España: Síntesis.

PERKINS, R. y RINALDI, M. (2002). Unemployment rates among patients with long-term mental health problems. A decade of rising unemployment. Psychiatric Bulletin, 26, 295-298, https://doi.org/10.11 92/pb.26.8.295

MÉNDEZ GARCÍA, M. Á. (2010). Estudio psicométrico de una escala etnopsicológica de deseabilidad social para el contexto mexicano (Doctoral dissertation, Universidad Iberoamericana). 
MÉTODO ISTAS (CoPsoQ), instrumento para la prevención de riesgos psicosociales, versión corta para pequeñas empresas y autoevaluación (Versión 21) (Armangué, Jorgensen y Schaltz, 2003).

MORENO JIMÉNEZ, B. y Báez León, C. (2010). Factores y riesgos psicosociales, formas, consecuencias, medidas y buenas prácticas. España: Universidad Autónoma de Madrid.

MORENO JIMÉNEZ, B. \& Garrosa Hernández, E. (2013). Salud laboral. Ediciones Pirámide.

OIT (1998). Enciclopedia de salud y seguridad en el trabajo. 3aed. España: OIT-INSHT.

PARRA, M. (2003). Conceptos básicos en salud laboral. Santiago de Chile: Oficina Internacional del Trabajo, OIT.

RENDA, E., Rozas Garay, M., Moscardini, O. y Torchia, N. M. (2017). In Ministerio de Seguridad de la Nación (Ed.), Manual para la elaboración de mapas de riesgo (1ํㅡㄹ. ed.). Argentina: Programa Naciones Unidas para el Desarrollo PNUD.

RIVERA-PORRAS, D. (2019) Gestión del riesgo psicosocial y organizacional, un análisis bibliométrico. Aibi revista de investigación, administración e ingeniería, 1(7), pp. 26-30.

SALAMANCA, S.R., Pérez, J.M., Infante, A.F., Olarte, Y.Y. (2019). Análisis de los factores de riesgo psicosocial a nivel nacional e internacional. Revista TEMAS, III (13), 39-45.

SCHEIN, E. H. (1988). La cultura empresarial y el liderazgo. Barcelona: Plaza y Janés.

SOBRINO, T. (2001). La inserción laboral de la persona con enfermedad mental crónica. Boletín AMRP, 8(13), 13-23. 